\title{
Monodromy, Duality and Integrability of Two Dimensional String Effective Action
}

\author{
Ashok Das*†, J. Maharana ${ }^{\ddagger}$ and A. Melikyan ${ }^{\dagger}$ \\ $\dagger$ Department of Physics and Astronomy \\ University of Rochester, Rochester, NY 14627-0171, USA. \\ E-mail: 'das@pas.rochester edu, arsen@pas.rochester.edu' \\ $\ddagger$ Institute of Physics \\ Bhubaneswar -751005, India \\ E-mail: 'maharana@iopb.res.in
}

ABSTRACT: In this talk, we show how the monodromy matrix, $\widehat{\mathcal{M}}$, can be constructed for the two dimensional tree level string effective action. The pole structure of $\widehat{\mathcal{M}}$ is derived using its factorizability property. It is shown that the monodromy matrix transforms non-trivially under the non-compact T-duality group, which leaves the effective action invariant and this can be used to construct the monodromy matrix for more complicated backgrounds starting from simpler ones. We construct, explicitly, $\widehat{\mathcal{M}}$ for the exactly solvable Nappi-Witten model, both when $B=0$ and $B \neq 0$, where these ideas can be directly checked.

\section{Introduction}

The field theories in two space-time dimensions have attracted considerable attention over the past few decades. They possess a variety of interesting features. Some of these field theories, capture several salient characteristics of four dimensional theories and, therefore, such two dimensional models are used as theoretical laboratories. Moreover, the nonperturbative properties of field theories are much simpler to study in two dimensional models. There are classes of two dimensional theories which are endowed with a rich symmetry

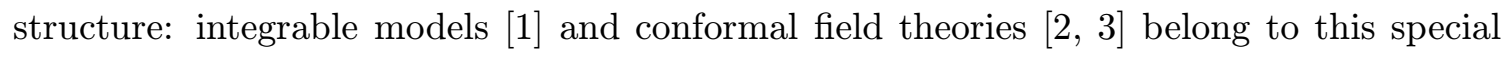

\footnotetext{
${ }^{*}$ Speaker.
} 
category among others. Under special circumstances, in the presence of isometries, a four dimensional theory may also be described by an effective two dimensional theory.

The string theories are abundantly rich in their symmetry content. The tree level string effective action, dimensionally reduced to lower dimensions, is known to possess en-

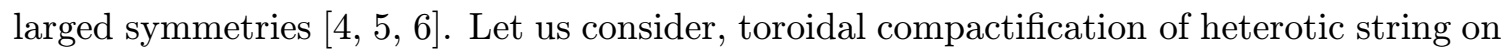
a $d$-dimensional torus, from 10 -dimensional space-time to $10-d$ dimensions. The reduced theory is known to be invariant under the non-compact T-duality group, $O(d, d+16)$. For the case $d=6$, namely, in the case of reduction to four space-time dimensions, the field strength of the two form anti-symmetric tensor can be traded for the pseudoscalar axion. Furthermore, the dilaton and axion can be combined to parameterize the coset $\frac{S L(2, R)}{U(1)}$. Thus, the four dimensional theory possesses the T-duality as well as the S-duality group of symmetries. When the string effective action is reduced to two space-time dimensions we

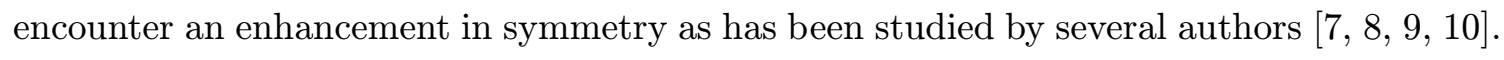
The string effective action describes supergravity theories and the integrability properties of such theories have been investigated in the recent past [1 $12 \overline{2}, ', \overline{1} \overline{3}_{1}$. It is worth while to mention that higher dimensional Einstein theory, dimensionally reduced to effective two dimensional theories, has been studied in the past [1] the monodromy matrix which encodes some of the essential features of integrable field theories. An effective two dimensional action naturally appears when one considers some aspects of black hole physics, colliding plane waves as well as special types of cosmological models. Recently, we have shown that the two dimensional string effective action has connection with integrable systems from a new perspective in the sense that one can construct the monodromy matrix for such theories with well defined prescriptions [i1 $\left.{ }_{1}^{1} \overline{1}\right]$. It was shown, while investigating the collision of plane fronted stringy waves, that the monodromy matrix can be constructed explicitly for a given set of background configurations [i] $\left.\overline{6}_{-1}\right]$. Subsequently, we were able to give the procedures for deriving the monodromy matrix under general settings. It is worth while to mention that some of the interesting aspects of black hole physics can be described by an effective two dimensional theory [1] there is an intimate relation between colliding plane waves and the description of four dimensional space-time with two commuting Killing vectors.

Chandrasekhar and Xanthopoulos [22] in their seminal work, have shown that a violently time dependent space-time with a pair of Killing vectors provides a description of plane colliding gravitational waves. Furthermore, Ferrari, Ibanez and Bruni [2]23i] have demonstrated that the colliding plane wave metric can be identified, locally, to be isometric to the interior of a Schwarzschild metric. In another important step, Yurtsever [2] constructed the transformation which provides a connection between a metric describing colliding waves and one corresponding to the Schwarzschild black hole. As is well known, from the study of colliding plane gravitational waves [2흘 corresponding to massless states of strings, there is a curvature singularity in the future. Recently, the appearance of the future singularity, has found an alternative description in the context of Pre-Big Bang (PBB) scenario $\left[\overline{2} \overline{2}_{1}^{\prime}, \overline{2} \overline{2} \overline{7}\right]$. The incoming plane waves are to be identified as the initial stringy vacuua of the Universe, which collide and lead to the creation of the Universe. Indeed, the solutions correspond to Kasner type metric and the exponents fulfill the requirements of 
PBB conditions. A very important fact, in this context, is that one starts from a four dimensional effective action; however, the physical process is effectively described by a two dimensional theory.

When we focus our attention to address these problems in the frame work of string theory, it is essential to keep in mind the special symmetries, such as dualities, which are an integral part of the stringy symmetries. We have investigated [i] odromy matrix under T-duality transformation of the backgrounds under a general setting when the two dimensional action is derived from a $D$-dimensional string effective action through compactification on a $d$-dimensional torus, $T^{d}$. It was shown that the monodromy matrix transforms non-trivially under the duality group $O(d, d)$. Therefore, it opens up the possibility of studying integrable systems which might appear in the context of string theories. As an example, we considered the Nappi-Witten [i] $[\overline{9}]$ model, which is a solution to an exact conformal field theory described by a WZW model. We first obtained the monodromy matrix for the case when the 2-form anti-symmetric tensor is set to zero. It is also well known that an anti-symmetric tensor background can be generated through an $O(2,2)$ transformation from the initial backgrounds [i2 $\overline{2} \overline{8}]$. We can construct the monodromy matrix for the new set of backgrounds using our prescriptions. On the other hand, the new monodromy matrix can be constructed directly by utilizing the transformation rules discovered by us. Indeed, we explicitly, demonstrated that the monodromy matrices obtained via the two different routes are identical. It is obvious from the preceding discussions that the symmetries of the effective action play an important role in the construction of the monodromy matrix and its transformation properties under those symmetry transformations. Therefore, it is natural to expect intimate connections between the integrability properties of the two dimensional theory and the full stringy symmetry groups of $\mathrm{T}$ and $\mathrm{S}$ dualities.

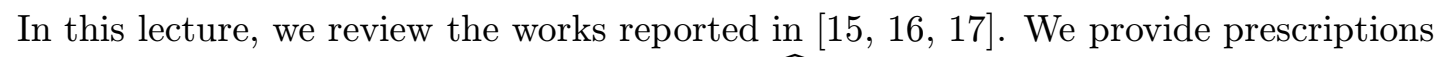
for the construction of the monodromy matrix, $\widehat{\mathcal{M}}$, for the string theoretic two dimensional effective action. We present the pole structure of the monodromy matrix from general arguments. The duality transformation properties of $\widehat{\mathcal{M}}$ follow from the definition and construction of this matrix. For example, if the action respects T-duality, then one can derive how $\widehat{\mathcal{M}}$ transforms under the group, whereas, if the underlying symmetry corresponds to S-duality an appropriate transformation rule for monodromy matrix can also be derived. Our paper is organized as follows: In Section II, we recapitulate the form of the two dimensional action obtained by toroidal compactification from higher dimensions. Then, we present the equations of motion. A key ingredient, in the derivation of the monodromy matrix, is the coset space reformulation of the reduced action. We devote Section III to the construction of the monodromy matrix for the problem under consideration. The transformation rules for $\widehat{\mathcal{M}}$, under a T-duality transformation, are derived once the matrix is constructed. An interesting observation is that the expression for $\widehat{\mathcal{M}}$ already captures the stringy symmetry in an elegant manner. In this section, we also present explicit forms of $\widehat{\mathcal{M}}$ for simple background configurations which still preserve some of the general features. We present an illustrative example in Section IV, namely, the structure of the Nappi-Witten model in the present context is analyzed in detail. We present a brief conclusion in Section V. 


\section{Two dimensional effective action}

In this section, we will briefly recapitulate the form of the string effective action in two dimensions, which will form the basis for all our subsequent discussions. Let us consider, for simplicity, the tree level string effective action in $D$-dimensions consisting of the graviton, the dilaton and the anti-symmetric tensor field,

$$
\hat{S}=\int d^{D} x \sqrt{-\hat{G}} e^{-\hat{\phi}}\left(R_{\hat{G}}+(\hat{\partial} \hat{\phi})^{2}-\frac{1}{12} \hat{H}_{\hat{\mu} \hat{\nu} \hat{\rho}} \hat{H}^{\hat{\mu} \hat{\nu} \hat{\rho}}\right)
$$

Here, $\hat{G}_{\hat{\mu} \hat{\nu}}$ is the $D$-dimensional metric in the string frame with signature $(-,+, \cdots,+)$, and $\hat{G}=\operatorname{det} \hat{G}_{\hat{\mu} \hat{\nu}} . \hat{R}_{\hat{G}}$ is the scalar curvature, $\hat{\phi}$ is the dilaton and $\hat{H}_{\hat{\mu} \hat{\nu} \hat{\rho}}=\partial_{\hat{\mu}} \hat{B}_{\hat{\nu} \hat{\rho}}+\partial_{\hat{\rho}} \hat{B}_{\hat{\mu} \hat{\nu}}+\partial_{\hat{\nu}} \hat{B}_{\hat{\rho} \hat{\mu}}$ is the field strength for the second-rank anti-symmetric tensor field, $\hat{B}_{\hat{\mu} \hat{\nu}}$.

If we compactify this action on a $d$-dimensional torus, $T^{d}$, where $d=D-2$, then, the resulting dimensionally reduced action would describe the two dimensional string effective action, which has the form [i2 $\overline{2} \overline{0}, \overline{2}, \overline{2} \overline{1}]$,

$$
S=\int d x^{0} d x^{1} \sqrt{-g} e^{-\bar{\phi}}\left(R+(\partial \bar{\phi})^{2}+\frac{1}{8} \operatorname{Tr}\left(\partial_{\alpha} M^{-1} \partial^{\alpha} M\right)\right)
$$

Here $\alpha, \beta=0,1$ are the two-dimensional space-time indices, $g_{\alpha \beta}$ is the two-dimensional space-time metric with $g=\operatorname{det} g_{\alpha \beta} . R$ is the corresponding two dimensional scalar curvature, while the shifted dilaton is defined as

$$
\bar{\phi}=\phi-\frac{1}{2} \log \operatorname{det} G_{i j}
$$

where $G_{i j}$ is the metric in the internal space, corresponding to the toroidally compactified coordinates $x^{i}, i, j=2,3, \ldots, D-1(d=D-2)$. Finally, $M$ is a $2 d \times 2 d$ symmetric matrix of the form

$$
M=\left(\begin{array}{cc}
G^{-1} & -G^{-1} B \\
B G^{-1} & G-B G^{-1} B
\end{array}\right)
$$

where $B$ represents the moduli coming from the dimensional reduction of the $\hat{B}$-field in $D$-dimensions. In general, there will be additional terms in (2.2i) associated with $d$ Abelian gauge fields arising from the metric, $\hat{G}_{\hat{\mu} \hat{\nu}}$, and another set of $d$ Abelian gauge fields coming from the anti-symmetric tensor, $\hat{B}_{\hat{\mu} \hat{\nu}}$, as a result of dimensional reduction [20 $\left.\overline{0}_{1}^{\prime}\right]$. Furthermore, there would also have been terms involving the field strength of the two dimensional tensor field, $B_{\alpha \beta}$. Since we are in two space-time dimensions, we have dropped the gauge field terms and, in the same spirit, have not kept the field strength of $B_{\alpha \beta}$, which can always be removed, if it depends only on the coordinates $x^{0}$ and $x^{1}$. Later, we will comment on the gauge fields, which assume a significant role, when Abelian gauge fields are present in the original string effective action $(\overline{2} \cdot \overline{2} .1)$.

The matrix $M$ corresponds to a symmetric representation of the group $O(d, d)$ and the dimensionally reduced action in $(\overline{2} \cdot \overline{2} 2)$ is invariant under the global $O(d, d)$ transformations

$$
\begin{gathered}
g_{\alpha \beta} \rightarrow g_{\alpha \beta}, \quad \bar{\phi} \rightarrow \bar{\phi} \\
M \rightarrow \Omega^{T} M \Omega
\end{gathered}
$$


where $\Omega \in O(d, d)$ is the global transformation matrix, which preserves the $O(d, d)$ metric

$$
\eta=\left(\begin{array}{cc}
0 & \mathbf{1}_{d} \\
\mathbf{1}_{d} & 0
\end{array}\right)
$$

with $\mathbf{1}_{d}$ representing the identity matrix in $d$ dimensions. Namely, $\Omega$ satisfies $\Omega^{T} \eta \Omega=\eta$.

The equations of motion for the different fields follow from the dimensionally reduced effective action $(\overline{2} \cdot \overline{2} \cdot \overline{2})$. For example, varying the effective action $(\overline{2} . \overline{2} i)$ with respect to the shifted dilaton, $\bar{\phi}$, and the metric, $g_{\alpha \beta}$, leads respectively to

$$
\begin{aligned}
R+2 g^{\alpha \beta} D_{\alpha} D_{\beta} \bar{\phi}-g^{\alpha \beta} \partial_{\alpha} \bar{\phi} \partial_{\beta} \bar{\phi}+\frac{1}{8} g^{\alpha \beta} \operatorname{Tr}\left(\partial_{\alpha} M^{-1} \partial_{\beta} M\right) & =0 \\
R_{\alpha \beta}+D_{\alpha} D_{\beta} \bar{\phi}+\frac{1}{8} \operatorname{Tr}\left(\partial_{\alpha} M^{-1} \partial_{\beta} M\right) & =0
\end{aligned}
$$

It follows from these that

$$
D_{\alpha} D^{\alpha} e^{-\bar{\phi}}=0
$$

The variation of the effective action with respect to $M$ needs some care since $M$ is a symmetric $O(d, d)$ matrix satisfying $M \eta M=\eta$. A simple method, for example, would involve adding the constraint to the effective action through a Lagrange multiplier. In any case, since there is no potential term in the effective action involving the matrix $M$, the Euler-Lagrange equation of motion following from the variation of the action with respect to $M$ has the form of a conservation law

$$
\partial_{\alpha}\left(e^{-\bar{\phi}} \sqrt{-g} g^{\alpha \beta} M^{-1} \partial_{\beta} M\right)=0
$$

We can further simplify these equations by working in the light-cone coordinates

$$
x^{+}=\frac{1}{\sqrt{2}}\left(x^{0}+x^{1}\right), \quad x^{-}=\frac{1}{\sqrt{2}}\left(x^{0}-x^{1}\right)
$$

and choosing the conformal gauge for the two dimensional metric, namely,

$$
g_{\alpha \beta}=e^{F\left(x^{+}, x^{-}\right)} \eta_{\alpha \beta}
$$

In this case, eqs. $\left(\overline{2}_{2}^{2} . \overline{1}_{1}^{1}\right)$ and $\left(\overline{2} . \overline{1} \overline{1}_{1}\right)$ take respectively the forms

$$
\begin{aligned}
\partial_{+} \partial_{-} e^{-\bar{\phi}} & =0 \\
\partial_{+}\left(e^{-\bar{\phi}} M^{-1} \partial_{-} M\right)+\partial_{-}\left(e^{-\bar{\phi}} M^{-1} \partial_{+} M\right) & =0
\end{aligned}
$$

while eqs. $\left(\overline{2} . \bar{z}_{1}^{i}\right)$ and $\left(\overline{2}_{2} . \overline{9}_{1}^{i}\right)$ can be written explicitly as

$$
\begin{aligned}
\partial_{+}^{2} \bar{\phi}-\partial_{+} F \partial_{+} \bar{\phi}+\frac{1}{8} \operatorname{Tr}\left(\partial_{+} M^{-1} \partial_{+} M\right) & =0 \\
\partial_{+} \partial_{-} \bar{\phi}-\partial_{+} \partial_{-} F+\frac{1}{8} \operatorname{Tr}\left(\partial_{+} M^{-1} \partial_{-} M\right) & =0 \\
\partial_{-}^{2} \bar{\phi}-\partial_{-} F \partial_{-} \bar{\phi}+\frac{1}{8} \operatorname{Tr}\left(\partial_{-} M^{-1} \partial_{-} M\right) & =0
\end{aligned}
$$


It is well known [20 $]$ ] that the moduli appearing in the definition of the $M$ matrix (see eq. $\left.\left(\overline{2}_{2} . \bar{l}_{i}\right)\right)$ parameterize the coset $\frac{O(d, d)}{O(d) \times O(d)}$. Correspondingly, it is convenient to introduce a triangular matrix $V \in \frac{O(d, d)}{O(d) \times O(d)}$, of the form

$$
V=\left(\begin{array}{cc}
E^{-1} & 0 \\
B E^{-1} & E^{T}
\end{array}\right)
$$

such that $M=V V^{T}$. Here, $E$ is the vielbein in the internal space so that $\left(E^{T} E\right)_{i j}=G_{i j}$. Under a combined global $O(d, d)$ and a local $O(d) \times O(d)$ transformation

$$
V \longrightarrow \Omega^{T} V h(x)
$$

where $\Omega \in O(d, d)$ and $h(x) \in O(d) \times O(d)$,

$$
M=V V^{T} \longrightarrow \Omega^{T} M \Omega
$$

Namely, the $M$ matrix is sensitive only to a global $O(d, d)$ rotation.

From the matrix $V$, we can construct the current $V^{-1} \partial_{\alpha} V$, which belongs to the Lie algebra of $O(d, d)$ and can be decomposed as

$$
V^{-1} \partial_{\alpha} V=P_{\alpha}+Q_{\alpha}
$$

Here, $Q_{\alpha}$ belongs to the Lie algebra of the maximally compact subgroup $O(d) \times O(d)$ and $P_{\alpha}$ belongs to the complement. Furthermore, it follows from the symmetric space automorphism property of the coset $\frac{O(d, d)}{O(d) \times O(d)}$ that $P_{\alpha}^{T}=P_{\alpha}, Q_{\alpha}^{T}=-Q_{\alpha}$ so that we can identify

$$
\begin{aligned}
P_{\alpha} & =\frac{1}{2}\left(V^{-1} \partial_{\alpha} V+\left(V^{-1} \partial_{\alpha} V\right)^{T}\right) \\
Q_{\alpha} & =\frac{1}{2}\left(V^{-1} \partial_{\alpha} V-\left(V^{-1} \partial_{\alpha} V\right)^{T}\right)
\end{aligned}
$$

It is now straightforward to check that

$$
\operatorname{Tr}\left(\partial_{\alpha} M^{-1} \partial_{\beta} M\right)=-4 \operatorname{Tr}\left(P_{\alpha} P_{\beta}\right)
$$

Furthermore, under a global $O(d, d)$ rotation, the currents in $(2 . \overline{2} 1)$ are invariant, while under a local $O(d) \times O(d)$ transformation, $V \longrightarrow V h(x)$,

$$
P_{\alpha} \longrightarrow h^{-1}(x) P_{\alpha} h(x), \quad Q_{\alpha} \longrightarrow h^{-1}(x) Q_{\alpha} h(x)+h^{-1}(x) \partial_{\alpha} h(x)
$$

Namely, under a local $O(d) \times O(d)$ transformation, $Q_{\alpha}$ transforms like a gauge field, while $P_{\alpha}$ transforms as belonging to the adjoint representation. It is clear, therefore, that (2.2.2 is invariant under the global $O(d, d)$ as well as the local $O(d) \times O(d)$ transformations. Consequently, the action in (2.2.2) is also invariant under the local $O(d) \times O(d)$ transformations.

This brings out, naturally, the connection between the system under study and two dimensional integrable systems. First, let us note that, in the absence of gravity and the dilaton (namely, if $g_{\alpha \beta}=\eta_{\alpha \beta}, \bar{\phi}=0$ ), the action in (2.2.2i) simply corresponds to a flat 
space sigma model defined over the coset $\frac{O(d, d)}{O(d) \times O(d)}$, which can be analyzed through a zero curvature condition with a constant spectral parameter (to be discussed in more detail in the next section). In the presence of gravity as well as the dilaton, we can eliminate the dilaton from the action (2.2.i) by choosing the particular conformal gauge $g_{\alpha \beta}=e^{\bar{\phi}} \eta_{\alpha \beta}$. In this case, the action will describe a sigma model, defined over the coset $\frac{O(d, d)}{O(d) \times O(d)}$, coupled to gravity. As we will show in the next section, this system can also be analyzed through a zero curvature condition much like the flat space case, although consistency requires the spectral parameter, in this case, to be space-time dependent.

So far, we have only discussed the two dimensional string effective action starting from the $D$-dimensional action in $\left(\overline{2} \cdot \overline{2}_{1}^{\prime}\right)$ involving the graviton, the dilaton and the antisymmetric tensor field. However, the action in ( $\left(\overline{2} . \overline{1}_{1}\right)$ can be generalized by adding $n$ Abelian gauge fields, with the additional action of the form (such terms naturally arise in heterotic string theory)

$$
\hat{S}_{\hat{A}}=-\frac{1}{4} \int d^{D} x \sqrt{-\hat{G}} e^{-\hat{\phi}}\left(\hat{g}^{\hat{\mu} \hat{\rho}} \hat{g} \hat{\nu} \hat{\lambda} \delta_{I J} \hat{F}_{\hat{\mu} \hat{\nu}}^{I} \hat{F}_{\hat{\rho} \hat{\lambda}}^{J}\right)
$$

where $I, J=1,2, \ldots, n$ and

$$
\hat{F}_{\hat{\mu} \hat{\nu}}^{I}=\partial_{\hat{\mu}} \hat{A}_{\hat{\nu}}^{I}-\partial_{\hat{\nu}} \hat{A}_{\hat{\mu}}^{I}
$$

This action can also be dimensionally reduced [20 201$]$ to two dimensions and the resulting effective action takes the form

$$
S_{A}=-\frac{1}{4} \int d x^{0} d x^{1} \sqrt{-g} e^{-\bar{\phi}}\left(F_{\alpha \beta}^{I} F^{I \alpha \beta}+2 F_{\alpha j}^{I} F^{I \alpha j}\right)
$$

where we have defined

$$
\begin{aligned}
a_{i}^{I} & =\hat{A}_{i}^{I} \\
A_{\alpha}^{(1) I} & =\hat{G}_{\alpha}^{I} \\
A_{\alpha}^{(3) I} & =\hat{A}_{\alpha}^{I}-a_{j}^{I} A_{\alpha}^{(1) j} \\
F_{\alpha \beta}^{(1) i} & =\partial_{\alpha} A_{\beta}^{(1) i}-\partial_{\beta} A_{\alpha}^{(1) i} \\
F_{\alpha \beta}^{(3) I} & =\partial_{\alpha} A_{\beta}^{(3) I}-\partial_{\beta} A_{\alpha}^{(3) I} \\
F_{\alpha \beta}^{I} & =F_{\alpha \beta}^{(3) I}+F_{\alpha \beta}^{(1) i} a_{i}^{I} \\
F_{\alpha i}^{I} & =\partial_{\alpha} a_{i}^{I}
\end{aligned}
$$

In the presence of the Abelian gauge fields, the field strength, $H$, associated with the second rank anti-symmetric tensor field, $B$, needs to be redefined for gauge invariance as

$$
\begin{aligned}
& H_{\alpha i j}=\partial_{\alpha} B_{i j}+\frac{1}{2}\left(a_{i}^{I} \partial_{\alpha} a_{j}^{I}-a_{j}^{I} \partial_{\alpha} a_{i}^{I}\right) \\
& H_{\alpha \beta i}=-C_{i j} F_{\alpha \beta}^{(1) j}+F_{\alpha \beta i}^{(2)}-a_{i}^{I} F_{\alpha \beta}^{(3) I} \\
& H_{\alpha \beta \gamma}=\partial_{\alpha} B_{\beta \gamma}-\frac{1}{2} \mathcal{A}_{\alpha}^{r} \eta_{r s} \mathcal{F}_{\beta \gamma}^{s}+\text { cyc. perms. }
\end{aligned}
$$


where $\mathcal{A}_{\alpha}^{r}=\left(A_{\alpha}^{(1) i}, A_{\alpha i}^{(2)}, A_{\alpha}^{(3) I}\right), \mathcal{F}_{\alpha \beta}^{r}=\partial_{\alpha} \mathcal{A}_{\beta}^{r}-\partial_{\beta} \mathcal{A}_{\alpha}^{r}$ and

$$
\begin{aligned}
A_{\alpha i}^{(2)} & =\hat{B}_{\alpha i}+B_{i j} A_{\alpha}^{(1) j}+\frac{1}{2} a_{i}^{I} A_{\alpha}^{(3) I} \\
F_{\alpha \beta i}^{(2)} & =\partial_{\alpha} A_{\beta i}^{(2)}-\partial_{\beta} A_{\alpha i}^{(2)} \\
C_{i j} & =\frac{1}{2} a_{i}^{I} a_{j}^{I}+B_{i j}
\end{aligned}
$$

Once again, it is easy to see that, in two space-time dimensions, the field strength $H_{\alpha \beta \gamma}$ can be set to zero. Furthermore, keeping all other terms, the complete two dimensional string effective action can be shown to have the same form as in $(\overline{2} . \overline{2} \cdot \bar{i})$ with

$$
M=\left(\begin{array}{ccc}
G^{-1} & -G^{-1} C & -G^{-1} a^{T} \\
-C G^{-1} & G+C^{T} G^{-1} C & C^{T} G^{-1} a^{T}+a^{T} \\
-a G^{-1} & a G^{-1} C+a & 1+a G^{-1} a^{T}
\end{array}\right)
$$

In this case, $M$ is a symmetric $d \times(d+n)$ matrix $(d=D-2)$ belonging to $O(d, d+n)$. Under a $O(d, d+n)$ transformation

$$
M \longrightarrow \Omega^{T} M \Omega
$$

where the parameter of transformation $\Omega \in O(d, d+n)$ satisfying $\Omega^{T} \eta \Omega=\eta$, where

$$
\eta=\left(\begin{array}{ccc}
0 & \mathbf{1}_{d} & 0 \\
\mathbf{1}_{d} & 0 & 0 \\
0 & 0 & \mathbf{1}_{n}
\end{array}\right)
$$

represents the metric for $O(d, d+n)$. As in the earlier case, it is more convenient to introduce a matrix $V \in \frac{O(d, d+n)}{O(d) \times O(d+n)}$ of the form

$$
V=\left(\begin{array}{ccc}
E^{-1 T} & 0 & 0 \\
-C^{T} E^{-1 T} & E^{T} & a^{T} \\
-a E^{-1 T} & 0 & 1
\end{array}\right)
$$

such that $M=V V^{T}$. As before, under a combined global $O(d, d+n)$ and a local $O(d) \times$ $O(d+n)$ transformation

$$
V \longrightarrow \Omega^{T} V h(x)
$$

where $\Omega \in O(d, d+n)$ and $h(x) \in O(d) \times O(d+n)$. However, the matrix $M$ is not sensitive to the local $O(d) \times O(d+n)$ transformations. We can now define the current $V^{-1} \partial_{\alpha} V$ which belongs to the Lie algebra of $O(d, d+n)$ and which can be decomposed as

$$
V^{-1} \partial_{\alpha} V=P_{\alpha}+Q_{\alpha}
$$

In the present case, $Q_{\alpha}$ belongs to the Lie algebra of the maximal compact subgroup $O(d) \times$ $O(d+n)$, while $P_{\alpha}$ belongs to the complement. Under a global $O(d, d+n)$ transformation, $P_{\alpha}$ and $Q_{\alpha}$ are invariant, while under a local $O(d) \times O(d+n)$ transformation, $V \rightarrow V h(x)$,

$$
P_{\alpha} \longrightarrow h^{-1}(x) P_{\alpha} h(x), \quad Q_{\alpha} \rightarrow h^{-1}(x) Q_{\alpha} h(x)+h^{-1}(x) \partial_{\alpha} h(x)
$$

and all the discussion for the earlier case can again be carried through. 


\section{Monodromy Matrix}

In the last section, we saw that the two dimensional string effective action, dimensionally reduced from $D$-dimensions, has a natural description of a sigma model defined on a coset, coupled to gravity. In the case when there are no Abelian gauge fields present in the starting string action, the sigma model is defined on the coset $\frac{O(d, d)}{O(d) \times O(d)}$ where $d=D-2$. On the other hand, if $n$ Abelian gauge fields are present in the starting string action, the coset can be identified with $\frac{O(d, d+n)}{O(d) \times O(d+n)}$. In this section, we will further analyze the integrability properties of such a system and construct the monodromy matrix associated with the system.

Let us consider a general sigma model in flat space-time, defined on the coset $G / H$. The two cases of interest for us are when $G=O(d, d), H=O(d) \times O(d)$ and $G=O(d, d+n), H=$ $O(d) \times O(d+n)$. Let $V \in G / H$ and $M=V V^{T}$. Then, as we have noted in the last section, we can decompose the current $V^{-1} \partial_{\alpha} V$ belonging to the Lie algebra of $G$ as

$$
V^{-1} \partial_{\alpha} V=P_{\alpha}+Q_{\alpha}
$$

where $Q_{\alpha}$ belongs to the Lie algebra of $H$, while $P_{\alpha}$ belongs to the complement. The integrability condition, following from this, corresponds to the zero curvature condition

$$
\partial_{\alpha}\left(V^{-1} \partial_{\beta} V\right)-\partial_{\beta}\left(V^{-1} \partial_{\alpha} V\right)+\left[\left(V^{-1} \partial_{\alpha} V\right),\left(V^{-1} \partial_{\beta} V\right)\right]=0
$$

Explicitly, this equation gives

$$
\begin{aligned}
\partial_{\alpha} Q_{\beta}-\partial_{\beta} Q_{\alpha}+\left[Q_{\alpha}, Q_{\beta}\right]+\left[P_{\alpha}, P_{\beta}\right] & =0 \\
D_{\alpha} P_{\beta}-D_{\beta} P_{\alpha} & =0
\end{aligned}
$$

where we have defined

$$
D_{\alpha} P_{\beta}=\partial_{\alpha} P_{\beta}+\left[Q_{\alpha}, P_{\beta}\right]
$$

The equations of motion for the flat space sigma model (see eq. (2.11i))

$$
\eta^{\alpha \beta} \partial_{\alpha}\left(M^{-1} \partial_{\beta} M\right)=0
$$

can be rewritten in the form

$$
\eta^{\alpha \beta} D_{\alpha} P_{\beta}=0
$$

Let us next introduce a one parameter family of matrices $\hat{V}(x, t)$ where $t$ is a constant parameter (and not time), also known as the spectral parameter, such that $\hat{V}(x, t=0)=$ $V(x)$ and

$$
\hat{V}^{-1} \partial_{\alpha} \hat{V}=Q_{\alpha}+\frac{1+t^{2}}{1-t^{2}} P_{\alpha}+\frac{2 t}{1-t^{2}} \epsilon_{\alpha \beta} P^{\beta}
$$

Then, it is straightforward to check that the integrability condition

$$
\partial_{\alpha}\left(\hat{V}^{-1} \partial_{\beta} \hat{V}\right)-\partial_{\beta}\left(\hat{V}^{-1} \partial_{\alpha} \hat{V}\right)+\left[\left(\hat{V}^{-1} \partial_{\alpha} \hat{V}\right),\left(\hat{V}^{-1} \partial_{\beta} \hat{V}\right)\right]=0
$$

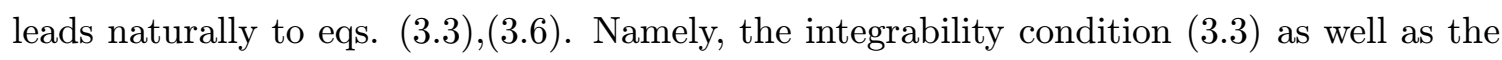
equation of motion for the flat space sigma model are obtained from the zero curvature condition associated with a potential which depends on a constant spectral parameter. 
In the presence of gravity, however, the equation for the sigma model modifies $[1,1 \overline{2}, 1,1 \overline{3}]$. In the conformal gauge $g_{\alpha \beta}=e^{\bar{\phi}} \eta_{\alpha \beta}$, eq. (2. $\overline{2}(\overline{1})$ ) takes the form

$$
\begin{aligned}
\eta^{\alpha \beta} \partial_{\alpha}\left(e^{-\bar{\phi}} M^{-1} \partial_{\beta} M\right) & =0 \\
\text { or, } \quad \eta^{\alpha \beta} D_{\alpha}\left(e^{-\bar{\phi}} P_{\beta}\right)=D_{\alpha}\left(e^{-\bar{\phi}} P^{\alpha}\right) & =0
\end{aligned}
$$

As before, we can introduce a one parameter family of potentials depending on a spectral parameter and with a decomposition of the form $\left(\overline{3} \cdot \bar{z}_{1}\right)$. However, in this case, it is easy to check that the zero curvature condition in $(\overline{3} . \overline{8})$ leads to the correct dynamical equation as well as the integrability condition provided the spectral parameter is space-time dependent and satisfies

$$
\partial_{\alpha} t=-\frac{1}{2} \epsilon_{\alpha \beta} \partial^{\beta}\left(e^{-\bar{\phi}}\left(t+\frac{1}{t}\right)\right)
$$

In the conformal gauge, as we have seen earlier in $(2.14)$, the shifted dilaton satisfies a simple equation. Therefore, defining

$$
\rho(x)=e^{-\bar{\phi}}
$$

we note that the solution following from the equation for the shifted dilaton can be written as

$$
\rho(x)=\rho_{+}\left(x^{+}\right)+\rho_{-}\left(x^{-}\right)
$$

With this, the solution to eq. (3.10i) can be written as

$$
t(x)=\frac{\sqrt{\omega+\rho_{+}}-\sqrt{\omega-\rho_{-}}}{\sqrt{\omega+\rho_{+}}+\sqrt{\omega-\rho_{-}}}
$$

where $\omega$ is the constant of integration, which can be thought of as a global spectral parameter. It is clear that the solutions in eq. (3.13) are double valued in nature.

There are several things to note from our discussion so far. First of all, the one parameter family of connections (currents) does not determine the potential $\hat{V}(x, t)$ uniquely, namely, $\hat{V}$ and $S(\omega) \hat{V}$, where $S(\omega)$ is a constant matrix, yield the same one parameter family of connections. Second, in the presence of the spectral parameter, the symmetric space automorphism can be generalized as

$$
\eta^{\infty}(\hat{V}(x, t))=\eta\left(\hat{V}\left(x, \frac{1}{t}\right)\right)=\left(\hat{V}^{-1}\left(x, \frac{1}{t}\right)\right)^{T}
$$

It can be shown, following from this, that

$$
\left(\hat{V}^{-1}\left(x, \frac{1}{t}\right) \partial_{\alpha} \hat{V}\left(x, \frac{1}{t}\right)\right)^{T}=-\hat{V}^{-1}(x, t) \partial_{\alpha} \hat{V}(x, t)
$$

Given these, let us define

$$
\mathcal{M}=\hat{V}(x, t) \hat{V}^{T}\left(x, \frac{1}{t}\right)
$$

It follows now, from eq. (3.15), that

$$
\partial_{\alpha} \mathcal{M}=0
$$


Namely, $\mathcal{M}=\mathcal{M}(\omega)$ and is independent of space-time coordinates. $\mathcal{M}(\omega)$ is known as the monodromy matrix for the system under study and encodes properties of integrability such as the conserved quantities associated with the system.

Let us next describe how the monodromy matrix is constructed for such systems. For simplicity, we will consider the action in (2.2.2), which describes a sigma model defined on the coset $\frac{O(d, d)}{O(d) \times O(d)}$. The other case can be studied in a completely analogous manner. To start with, let us set the anti-symmetric tensor field to zero, namely, $B=0$. In this case, we can write

$$
M^{(B=0)}=\left(\begin{array}{cc}
G^{-1} & 0 \\
0 & G
\end{array}\right), \quad V^{(B=0)}=\left(\begin{array}{cc}
E^{-1} & 0 \\
0 & E
\end{array}\right)
$$

Let us further assume that the matrix $E$ and, therefore, $G$ are diagonal, as is relevant in the study of colliding plane waves. Namely, let us parameterize

$$
\begin{aligned}
& E=\operatorname{diag} .\left(e^{\frac{1}{2}\left(\lambda+\psi_{1}\right)}, e^{\frac{1}{2}\left(\lambda+\psi_{2}\right)}, \cdots, e^{\frac{1}{2}\left(\lambda+\psi_{d}\right)}\right) \\
& G=\operatorname{diag} .\left(e^{\lambda+\psi_{1}}, e^{\lambda+\psi_{2}}, \cdots, e^{\lambda+\psi_{d}}\right)
\end{aligned}
$$

with $\sum_{i} \psi_{i}=0$ so that $\lambda=\frac{1}{d} \log \operatorname{det} G$, as adopted in [2휴]

In this case, it follows that (see $(\overline{2} 2 . \overline{2} \bar{i}))$

$$
\begin{aligned}
P_{\alpha} & =\frac{1}{2}\left(\left(V^{(B=0)}\right)^{-1} \partial_{\alpha} V^{(B=0)}+\left(\left(V^{(B=0)}\right)^{-1} \partial_{\alpha} V^{(B=0)}\right)^{T}\right)=\left(\begin{array}{cc}
-E^{-1} \partial_{\alpha} E & 0 \\
0 & E^{-1} \partial_{\alpha} E
\end{array}\right) \\
Q_{\alpha} & =\frac{1}{2}\left(\left(V^{(B=0)}\right)^{-1} \partial_{\alpha} V^{(B=0)}-\left(\left(V^{(B=0)}\right)^{-1} \partial_{\alpha} V^{(B=0)}\right)^{T}\right)=0
\end{aligned}
$$

so that we have

$$
\left(\hat{V}^{(B=0)}\right)^{-1} \partial_{+} \hat{V}^{(B=0)}=\frac{1-t}{1+t} P_{+}, \quad\left(\hat{V}^{(B=0)}\right)^{-1} \partial_{-} \hat{V}^{(B=0)}=\frac{1+t}{1-t} P_{-}
$$

Since $P_{ \pm}$are diagonal matrices and $\hat{V}^{(B=0)}(x, t=0)=V^{(B=0)}(x)$ is diagonal, it follows that we can write

$$
\hat{V}^{(B=0)}(x, t)=\left(\begin{array}{cc}
\bar{V}^{(B=0)}(x, t) & 0 \\
0 & \left(\bar{V}^{(B=0)}\right)^{-1}(x, t)
\end{array}\right)
$$

with $\bar{V}^{(B=0)}(x, t)$ a diagonal matrix of the form $\left(\bar{V}_{1}, \bar{V}_{2}, \cdots, \bar{V}_{d}\right)$. Let us assume that

$$
\bar{V}_{i}=\frac{t_{d+i}}{t_{i}} \frac{t-t_{i}}{t-t_{d+i}} E_{i}^{-1}, \quad i=1,2, \cdots, d
$$

where $t_{i}$ is the spectral parameter corresponding to the constant $\omega_{i}$. Clearly, for $t=0$, this leads to the diagonal elements of $V^{(B=0)}$. Furthermore, noting the form of $P_{ \pm}$in $(3.20 \overline{0})$ and recalling that the spectral parameters satisfy

$$
\partial_{ \pm} t=\frac{1 \mp t}{1 \pm t} \partial_{ \pm} \ln \rho, \quad \partial_{ \pm} t_{i}=\frac{1 \mp t_{i}}{1 \pm t_{i}} \partial_{ \pm} \ln \rho
$$


it is easy to verify that

$$
\bar{V}_{i}^{-1} \partial_{ \pm} \bar{V}_{i}=\partial_{ \pm} \ln E_{i}^{-1} \mp \frac{t}{1 \pm t} \partial_{ \pm} \ln \left(-\frac{t_{i}}{t_{d+i}}\right)=\frac{1 \mp t}{1 \pm t} \partial_{ \pm} \ln E_{i}^{-1}
$$

provided we identify $\left(t_{i}\right.$ and $t_{d+i}$ have opposite signatures following from the double valued nature of the solutions in $(\overline{3} . \overline{3}$ i $))$

$$
-\frac{t_{i}}{t_{d+i}}=E_{i}^{-2}
$$

In that case, we can write

$$
\left(\hat{V}^{(B=0)}\right)^{-1} \partial_{ \pm} \hat{V}^{(B=0)}=\frac{1 \mp t}{1 \pm t}\left(\begin{array}{cc}
-E^{-1} \partial_{ \pm} E & 0 \\
0 & E^{-1} \partial_{ \pm} E
\end{array}\right)=\frac{1 \mp t}{1 \pm t} P_{ \pm}
$$

Thus, we see that, in the present case,

$$
\bar{V}_{i}=\frac{t_{d+i}}{t_{i}} \frac{t-t_{i}}{t-t_{d+i}} E_{i}^{-1}=\sqrt{-\frac{t_{d+i}}{t_{i}}} \frac{t-t_{i}}{t-t_{d+i}}
$$

and the matrix $\hat{V}^{(B=0)}(x, t)$ has $2 d$ simple poles - one pair for every diagonal element $E_{i}$. Furthermore, it is simple to check from eq. (

$$
\frac{\omega-\omega_{i}}{\omega-\omega_{d+i}}=\frac{t_{d+i}}{t_{i}} \frac{t-t_{i}}{t-t_{d+i}} \frac{\frac{1}{t}-t_{i}}{\frac{1}{t}-t_{d+i}}
$$

so that we can determine the monodromy matrix to be of the form

$$
\widehat{\mathcal{M}}^{(B=0)}=\hat{V}^{(B=0)}(x, t)\left(\hat{V}^{B=0)}\right)^{T}\left(x, \frac{1}{t}\right)=\left(\begin{array}{cc}
\mathcal{M}(\omega) & 0 \\
0 & \mathcal{M}^{-1}(\omega)
\end{array}\right)
$$

where $\mathcal{M}(\omega)$ is diagonal with

$$
\mathcal{M}_{i}(\omega)=\bar{V}_{i}(x, t) \bar{V}_{i}\left(x, \frac{1}{t}\right)=-\frac{\omega-\omega_{i}}{\omega-\omega_{d+i}}
$$

We note that the double valued relation between the global and the local spectral parameters allows us to choose $\omega_{d+i}=-\omega_{i}$, in which case, we have

$$
\mathcal{M}_{i}(\omega)=\frac{\omega_{i}-\omega}{\omega_{i}+\omega}
$$

This determines the monodromy matrix for the case when $B=0$.

Let us note next that we are dealing with a sigma model, obtained through dimensional reduction of a higher dimensional tree level string effective action. Therefore, the symmetries present in the string theory, such as $T$ - duality, should be encoded in the monodromy matrix as well. For example, it is known that one can generate new backgrounds (of the string theory) starting from given ones through $T$-duality transformations. In particular, starting from a background where $B=0$, it is possible, in some models (such as the Nappi-Witten model), to generate backgrounds with $B \neq 0$ through a $T$-duality rotation. 
It is natural, therefore, to examine how the monodromy matrix transforms under such transformations, for, then, we can determine the monodromy matrix for more complicated backgrounds starting from simpler ones.

Let us note that the $T$-duality transformation, within the context of string theory (without Abelian gauge fields), corresponds to a global $O(d, d)$ rotation. Since the one parameter family of matrices $\hat{V}(x, t) \in \frac{O(d, d)}{O(d) \times O(d)}$ much like $V(x)=\hat{V}(x, t=0)$, it follows that under a global $O(d, d)$ rotation

$$
\begin{aligned}
\hat{V}(x, t) & \longrightarrow \Omega^{T} \hat{V}(x, t) \\
\widehat{\mathcal{M}}(\omega)=\hat{V}(x, t) \hat{V}^{T}\left(x, \frac{1}{t}\right) & \longrightarrow \Omega^{T} \hat{V}(x, t) \hat{V}^{T}\left(x, \frac{1}{t}\right) \Omega=\Omega^{T} \widehat{\mathcal{M}}(\omega) \Omega
\end{aligned}
$$

Let us also recall that, under a local $O(d) \times O(d)$ transformation, $\hat{V}(x, t) \rightarrow \hat{V}(x, t) h(x)$. Therefore, the only local transformations which will preserve the global nature of the monodromy matrix are the ones that do not depend on the local spectral parameter explicitly. We have already seen that the matrix $M=V V^{T}$ is only sensitive to the global $O(d, d)$ transformations even though $V(x)$ transforms non-trivially under a combined global $O(d, d)$ and a local $O(d) \times O(d)$ transformation. In a similar manner, $\widehat{\mathcal{M}}(\omega)$ is only sensitive to the global $O(d, d)$ rotation. We will check this explicitly in the case of the Nappi-Witten model in the next section. For the moment, let us note that this brings out an interesting connection between the integrability properties of the two dimensional string effective action and its $T$-duality properties, which can be used as a powerful tool in determining solutions.

For completeness, we record here the transformation properties of $\widehat{\mathcal{M}}$ under an infinitesimal $O(d, d)$ transformation. Let us denote

$$
\widehat{\mathcal{M}}=\left(\begin{array}{ll}
\widehat{\mathcal{M}}_{11} & \widehat{\mathcal{M}}_{12} \\
\widehat{\mathcal{M}}_{21} & \widehat{\mathcal{M}}_{22}
\end{array}\right)
$$

where each element represents a $d \times d$ matrix. Infinitesimally, we can write [2001]

$$
\Omega=\left(\begin{array}{cc}
1+X & Y \\
Z & 1+W
\end{array}\right)
$$

where the infinitesimal parameters of the transformation satisfy $Y^{T}=-Y, Z^{T}=-Z$ and $W=-X^{T}$. Under such an infinitesimal transformation, it follows from (3.3.

$$
\begin{aligned}
& \delta \widehat{\mathcal{M}}_{11}=\widehat{\mathcal{M}}_{11} X+X^{T} \widehat{\mathcal{M}}_{11}-Z \widehat{\mathcal{M}}_{12}+\widehat{\mathcal{M}}_{12} Z \\
& \delta \widehat{\mathcal{M}}_{12}=\widehat{\mathcal{M}}_{11} Y+X^{T} \widehat{\mathcal{M}}_{12}-Z \widehat{\mathcal{M}}_{22}-\widehat{\mathcal{M}}_{12} X^{T} \\
& \delta \widehat{\mathcal{M}}_{21}=-Y \widehat{\mathcal{M}}_{11}-X \widehat{\mathcal{M}}_{21}+\widehat{\mathcal{M}}_{21} X+\widehat{\mathcal{M}}_{22} Z \\
& \delta \widehat{\mathcal{M}}_{22}=\widehat{\mathcal{M}}_{21} Y-Y \widehat{\mathcal{M}}_{12}-X \widehat{\mathcal{M}}_{22}-\widehat{\mathcal{M}}_{22} X^{T}
\end{aligned}
$$

\section{Application}

The ideas presented in the earlier section can be applied to various physical systems. For example, if we are considering collision of plane-fronted waves, which correspond to 
massless states of closed strings, because of the isometries in the problem, this can be described effectively by a two dimensional theory and all our earlier discussions can be carried over [i] $\left.\overline{6}_{1}\right]$. In this section, we will discuss one other class of physical phenomena where our results can be explicitly verified and prove quite useful.

\subsection{The Nappi-Witten Model}

The Nappi-Witten model [1] $\left.{ }_{1}^{1} \overline{9}\right]$ is an example of a cosmological solution following from the string theory. Let us note that, to leading order in $\alpha^{\prime}$, the string tension, there are several solutions to the string equations following from $\left(\begin{array}{l}2 \\ 2\end{array} .13\right)$ that constitute exact CFT backgrounds. One of these solutions, studied by Nappi and Witten, corresponds to a gauged $\frac{S L(2, R)}{S O(1,1)} \times \frac{S U(2)}{U(1)}$ Wess-Zumino-Witten model and describes a closed expanding universe in $3+1$ dimensions. The backgrounds consist of the metric, the dilaton and the anti-symmetric tensor fields of the forms (Here, we identify $x^{0}=\tau$.)

$$
\begin{aligned}
d s^{2} & =-d \tau^{2}+d x^{2}+\frac{1}{1-\cos 2 \tau \cos 2 x}\left(4 \cos ^{2} \tau \cos ^{2} x d y^{2}+4 \sin ^{2} \tau \sin ^{2} x d z^{2}\right) \\
\phi & =-\frac{1}{2} \log (1-\cos 2 \tau \cos 2 x) \\
B_{12} & =-B_{21}=b=\frac{(\cos 2 \tau-\cos 2 x)}{(1-\cos 2 \tau \cos 2 x)}
\end{aligned}
$$

Here, we have set an arbitrary constant parameter appearing in the Nappi-Witten solution to zero for simplicity.

Note that the backgrounds do not depend on two of the coordinates, namely, $(y, z)$ and, consequently, following from our earlier discussions, the system has an $O(2,2)$ symmetry. It is known that these backgrounds can be obtained from a much simpler background, with a vanishing $B$ field, of the form

$$
\begin{aligned}
d s^{2} & =-d \tau^{2}+d x^{2}+\frac{1}{\tan ^{2} \tau} d y^{2}+\tan ^{2} x d z^{2} \\
\bar{\phi} & =-\log (\sin 2 \tau \sin 2 x) .
\end{aligned}
$$

through an $O(2,2)$ rotation. In the language of our earlier discussion, we note that we can write

$$
G^{B=0}=\left(\begin{array}{cc}
e^{\lambda^{(0)}+\psi^{(0)}} & 0 \\
0 & e^{\lambda^{(0)}-\psi^{(0)}}
\end{array}\right)=\left(\begin{array}{cc}
\xi_{1} & 0 \\
0 & \xi_{2}
\end{array}\right)
$$

with

$$
\xi_{1}=\exp \left(\lambda^{0}+\psi^{0}\right)=\frac{1}{\tan ^{2} \tau}, \quad \xi_{2}=\exp \left(\lambda^{0}-\psi^{0}\right)=\frac{1}{\tan ^{2} x}
$$

where the superscript " 0 " denotes the vanishing $B$ field. In this case, therefore, we have

$$
M^{(B=0)}=\left(\begin{array}{cc}
\left(G^{(B=0)}\right)^{-1} & 0 \\
0 & G^{(B=0)}
\end{array}\right)
$$

On the other hand, it is easy to check that we can write

$$
G^{(B)}=\left(\begin{array}{cc}
\frac{2 \xi}{1+\xi_{1} \xi_{2}} & 0 \\
0 & \frac{2 \xi_{2}}{1+\xi_{1} \xi_{2}}
\end{array}\right)
$$




$$
B=-\frac{1-\xi_{1} \xi_{2}}{1+\xi_{1} \xi_{2}} \epsilon=b \epsilon
$$

where $\epsilon$ is the $2 \times 2$ anti-symmetric matrix

$$
\epsilon=\left(\begin{array}{cc}
0 & 1 \\
-1 & 0
\end{array}\right)
$$

so that

$$
M^{(B)}=\left(\begin{array}{cc}
\left(G^{(B)}\right)^{-1} & -\left(G^{(B)}\right)^{-1} B \\
B\left(G^{(B)}\right)^{-1} & G^{(B)}-B\left(G^{(B)}\right)^{-1} B
\end{array}\right)
$$

It is now a simple matter to check that

$$
M^{(B)}=\Omega^{T} M^{(B=0)} \Omega
$$

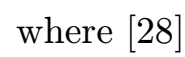

$$
\Omega=\frac{1}{\sqrt{2}}\left(\begin{array}{ll}
I & \epsilon \\
\epsilon & I
\end{array}\right)
$$

belongs to $O(2,2)$. Here, $I$ represents the $2 \times 2$ identity matrix while $\epsilon$ is the $2 \times 2$ antisymmetric matrix defined in $\left(\overline{4} \bar{t}_{1}\right)$. This shows that the backgrounds with a nontrivial anti-symmetric tensor field can be generated from a much simpler background with a vanishing $B$ field through a global $O(2,2)$ rotation.

It follows from eq. (4.

$$
E^{(B=0)}=\left(\begin{array}{cc}
\sqrt{\xi_{1}} & 0 \\
0 & \sqrt{\xi_{2}}
\end{array}\right)
$$

so that we have

$$
V^{(B=0)}=\left(\begin{array}{cc}
\left(E^{(B=0)}\right)^{-1} & 0 \\
0 & E^{(B=0)}
\end{array}\right)
$$

and it follows that (see eq. $\left(\overline{3} \cdot \overline{2} 0_{1}^{3}\right)$

$P_{ \pm}^{(B=0)}=\left(\begin{array}{cc}-\left(E^{(B=0)}\right)^{-1} \partial_{ \pm} E^{(B=0)} & 0 \\ 0 & \left(E^{(B=0)}\right)^{-1} \partial_{ \pm} E^{(B=0)}\end{array}\right)=\left(\begin{array}{cccc}-\frac{\left(\partial_{ \pm} \xi_{1}\right)}{\xi_{1}} & 0 & 0 & 0 \\ 0 & -\frac{\left(\partial_{ \pm} \xi_{2}\right)}{\xi_{2}} & 0 & 0 \\ 0 & 0 & \frac{\left(\partial_{ \pm} \xi_{1}\right)}{\xi_{1}} & 0 \\ 0 & 0 & 0 & \frac{\left(\partial_{ \pm} \xi_{2}\right)}{\xi_{2}}\end{array}\right)$

$Q_{ \pm}^{(B=0)}=0$

In this case, therefore, the one parameter family of potentials, $\hat{V}^{(B=0)}(x, t)$, have to satisfy (since $Q_{ \pm}^{(B=0)}=0$ )

$$
\left(\hat{V}^{(B=0)}\right)^{-1}(x, t) \partial_{ \pm} \hat{V}^{(B=0)}(x, t)=\frac{1 \mp t}{1 \pm t} P_{ \pm}^{(B=0)}
$$

where $t$ is the space-time dependent spectral parameter. 
Following our earlier construction (see $\left(3 . \overline{2} \overline{8}_{1}\right)$ ), we can determine

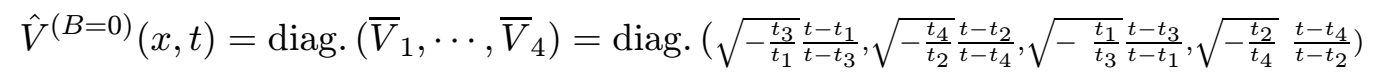

where

$$
-\frac{t_{1}}{t_{3}}=\left(E_{1}^{(B=0)}\right)^{-2}=\frac{1}{\xi_{1}}, \quad-\frac{t_{2}}{t_{4}}=\left(E_{2}^{(B=0)}\right)^{-2}=\frac{1}{\xi_{2}}
$$

It can be checked explicitly, using the equation satisfied by the spectral parameter, $(3.24)$, that $\hat{V}^{(B=0)}(x, t)$ in $(\overline{4} \cdot \overline{1})$ does indeed satisfy ( $\left(\overline{4}-\overline{1} \overline{1}_{1}\right)$. The monodromy matrix, in this case, follows to be

$$
\begin{aligned}
\widehat{\mathcal{M}}^{(B=0)} & =\left(\begin{array}{cc}
\mathcal{M}(\omega) & 0 \\
0 & \mathcal{M}^{-1}(\omega)
\end{array}\right)=\left(\begin{array}{cccc}
\frac{\omega-\omega_{1}}{\omega-\omega_{3}} & 0 & 0 & 0 \\
0 & \frac{\omega-\omega_{2}}{\omega-\omega_{4}} & 0 & 0 \\
0 & 0 & \frac{\omega-\omega_{3}}{\omega-\omega_{1}} & 0 \\
0 & 0 & 0 & \frac{\omega-\omega_{4}}{\omega-\omega_{2}}
\end{array}\right) \\
& =\left(\begin{array}{cccc}
\frac{\omega_{1}-\omega}{\omega_{1}+\omega} & 0 & 0 & 0 \\
0 & \frac{\omega_{2}-\omega}{\omega_{2}+\omega} & 0 & 0 \\
0 & 0 & \frac{\omega_{1}+\omega}{\omega_{1}-\omega} & 0 \\
0 & 0 & 0 & \frac{\omega_{2}+\omega}{\omega_{2}-\omega}
\end{array}\right)
\end{aligned}
$$

where we have identified $\omega_{3}=-\omega_{1}, \omega_{4}=-\omega_{2}$.

When $B \neq 0$, we can similarly obtain

$$
\begin{aligned}
E^{(B)} & =\left(\begin{array}{cc}
\sqrt{\frac{2 \xi_{1}}{1+\xi_{1} \xi_{2}}} & 0 \\
0 & \sqrt{\frac{2 \xi_{2}}{1+\xi_{1} \xi_{2}}}
\end{array}\right) \\
V^{(B)} & =\left(\begin{array}{cc}
\left(E^{(B)}\right)^{-1} & 0 \\
B\left(E^{(B)}\right)^{-1} & E^{(B)}
\end{array}\right)
\end{aligned}
$$

It follows now that

$$
\begin{aligned}
P_{ \pm}^{(B)} & =\left(\begin{array}{cc}
-\left(E^{(B)}\right)^{-1} \partial_{ \pm} E^{(B)} & -\frac{1}{2}\left(E^{(B)}\right)^{-1}\left(\partial_{ \pm} B\right)\left(E^{(B)}\right)^{-1} \\
\frac{1}{2}\left(E^{(B)}\right)^{-1}\left(\partial_{ \pm} B\right)\left(E^{(B)}\right)^{-1} & \left(E^{(B)}\right)^{-1} \partial_{ \pm} E^{(B)}
\end{array}\right) \\
Q_{ \pm} & =\left(\begin{array}{cc}
0 & \frac{1}{2}\left(E^{(B)}\right)^{-1}\left(\partial_{ \pm} B\right)\left(E^{(B)}\right)^{-1} \\
\frac{1}{2}\left(E^{(B)}\right)^{-1}\left(\partial_{ \pm} B\right)\left(E^{(B)}\right)^{-1} & 0
\end{array}\right)
\end{aligned}
$$

In this case, therefore, $Q_{ \pm} \neq 0$ and the one parameter family of potentials has to satisfy

$$
\left(\hat{V}^{(B)}\right)^{-1}(x, t) \partial_{ \pm} \hat{V}^{(B)}(x, t)=Q_{ \pm}^{(B)}+\frac{1 \mp t}{1 \pm t} P_{ \pm}^{(B)}
$$

It is straightforward to check that

$$
V^{(B)}(x)=\Omega^{T} V^{(B=0)}(x) h(x)
$$

where $\Omega$ is the $O(2,2)$ matrix defined in $(4.10)$ and $h(x) \in O(2) \times O(2)$ and is of the form

$$
h(x)=\frac{1}{\sqrt{2}}\left(\begin{array}{cc}
\sqrt{1-b} I & \sqrt{1+b} \epsilon \\
\sqrt{1+b} \epsilon & \sqrt{1-b} I
\end{array}\right)
$$


It can also be checked that

$$
\begin{gathered}
\hat{V}^{(B)}(x, t)=\Omega^{T} \hat{V}^{(B=0)}(x, t) h(x) \\
=\frac{1}{2}\left(\begin{array}{cccc}
\alpha \bar{V}_{1}+\beta \bar{V}_{4} & 0 & 0 & \beta \bar{V}_{1}-\alpha \bar{V}_{4} \\
0 & \alpha \bar{V}_{2}+\beta \bar{V}_{3} & -\beta \bar{V}_{2}+\alpha \bar{V}_{3} & 0 \\
0 & -\alpha \bar{V}_{2}+\beta \bar{V}_{3} & \beta \bar{V}_{2}+\alpha \bar{V}_{3} & 0 \\
\alpha \bar{V}_{1}-\beta \bar{V}_{4} & 0 & 0 & \beta \bar{V}_{1}+\alpha \bar{V}_{4}
\end{array}\right)
\end{gathered}
$$

where $\alpha=\sqrt{1-b}$ and $\beta=\sqrt{1+b}$, satisfies the defining relation in (4.20). We note here that,when $B \neq 0$, while $V^{(B)}(x)$ is triangular, $\hat{V}^{(B)}(x, t)$ is not in general and that both $V^{(B)}$ and $\hat{V}^{(B)}$ are related to their counterparts with $B=0$ through a combined global $O(2,2)$ and a local $O(2) \times O(2)$ transformation. Furthermore, as was pointed out earlier, the local transformation does not depend explicitly on the spectral parameter. This is quite crucial, for it immediately leads to

$$
\begin{aligned}
\widehat{\mathcal{M}}^{(B)} & =\hat{V}^{(B)}(x, t)\left(\hat{V}^{(B)}\right)^{T}\left(x, \frac{1}{t}\right)=\Omega^{T} \hat{V}^{(B=0)}(x, t) h(x) h^{T}(x)\left(\hat{V}^{(B=0)}\right)^{T}\left(x, \frac{1}{t}\right) \Omega=\Omega^{T} \widehat{\mathcal{M}}^{(B=0)} \Omega \\
& =\frac{1}{2}\left(\begin{array}{cccc}
\mathcal{M}_{1}+\mathcal{M}_{2}^{-1} & 0 & 0 & \mathcal{M}_{1}-\mathcal{M}_{2}^{-1} \\
0 & \mathcal{M}_{2}+\mathcal{M}_{1}^{-1} & -\mathcal{M}_{2}+\mathcal{M}_{1}^{-1} & 0 \\
0 & \mathcal{M}_{2}+\mathcal{M}_{1}^{-1} & \mathcal{M}_{2}+\mathcal{M}_{1}^{-1} & 0 \\
\mathcal{M}_{1}-\mathcal{M}_{2}^{-1} & 0 & 0 & \mathcal{M}_{1}+\mathcal{M}_{2}^{-1}
\end{array}\right)
\end{aligned}
$$

where $\mathcal{M}_{1}=\frac{\omega_{1}-\omega}{\omega_{1}+\omega}$ and $\mathcal{M}_{2}=\frac{\omega_{2}-\omega}{\omega_{2}+\omega}$ are the two diagonal elements of $\mathcal{M}(\omega)$ in (44.1 shows explicitly that, for backgrounds related by a duality transformation (in the present example, an $O(2,2)$ rotation), the corresponding monodromy matrices are also related in a simple manner, as was pointed out in the last section.

\section{Summary and Discussion}

In this talk, we have described the prescriptions for the construction of the monodromy matrix for two dimensional string effective action. We adopted the procedure commonly followed in the construction of the monodromy matrix for a class of two dimensional $\sigma$ models in curved space. As mentioned earlier, in most of the cases, the $\sigma$-model arises from the dimensional reduction of higher dimensional Einstein-Hilbert action to two dimensional space-time due to the presence of isometries. In the context of string theory, a similar approach was adopted in the past to construct the monodromy matrix as was the case with dimensionally reduced models in gravity.

One of our principal objective was to take into account the symmetries associated with the string effective action and construct the monodromy matrix which contains information about these symmetries. We have succeeded in introducing a procedure for the construction of the monodromy matrix under general grounds with some mild requirements such as factorizability and presence of isolated poles. Furthermore, we have demonstrated that the monodromy matrix transforms non-trivially under the non-compact T-duality group when the two dimensional string effective action respects that symmetry. We feel, this is an 
interesting and important result. The procedure, adopted by us, allows us to construct the monodromy matrix, once a set of string background configurations are known. As a result, if we know monodromy matrix for a given set of simple string vacuum backgrounds, we can directly obtain the corresponding monodromy matrix for another set of more complicated backgrounds, if the latter can be derived by duality transformations from the simpler backgrounds.

We have discussed an illustrative example in Section IV as an application of our methods. We considered the Nappi-Witten model which is exactly solvable for both vanishing and non-vanishing two form potential $B$. This is a good testing ground for duality transformation properties of the monodromy matrix. We have constructed this matrix for the case $B=0$. Subsequently, we have also constructed it for the case $B \neq 0$. Then, as a consistency check, we have derived the $\widehat{\mathcal{M}}^{B}$ from $\widehat{\mathcal{M}}^{B=0}$ following our rules of the transformations of $\widehat{\mathcal{M}}$ under duality. Indeed, it is found that the monodromy matrix computed using the two different ways, mentioned above, coincide. Although we have not discussed, the black hole solutions in heterotic string theory can also be described in this way [i] $\overline{1}$ ]. Namely, the monodromy matrix for the 'seed' Schwarzschild black hole in heterotic string theory can be constructed and that for charged black hole solutions can be obtained from this, since the T-duality transformations which generate charged black hole solutions are already known.

It is worth while to mention that all our results are derived for the case of classical two dimensional effective theory as is the case for effective two dimensional theories derived from higher dimensional Einstein-Hilbert action. It might be interesting to explore systematically the construction of the monodromy matrix and its properties in quantum theory. We hope the work presented here will find applications in diverse directions where one encounters effective two dimensional models in the context of string theory.

Acknowledgment: One of us (AD) would like to thank the organizers of the workshop on integrable models for the kind hospitality. This work is supported in part by US DOE Grant No. DE-FG 02-91ER40685.

\section{References}

[1] A. Das, Integrable Models, World Scientific, Singapore (1989); L. D. Faddeev, Integrable Models in (1+1)-dimensional Quantum Field Theory, Les Houches Lectures, 1982.

[2] E. Abdalla and M. C. B. Abdalla, Nonperturbative Methods in Two-dimensional Quantum Field Theory, World Scientific, Singapore(1991).

[3] M. B. Green, J. H. Schwarz and E. Witten, String theory, Cambridge University Press; J. Polchinski, String Theory, Cambridge University Press.

[4] A. Giveon, M. Porrati and E. Rabinovici, Phys. Rep. C244 (1994) 77, hep-th/9401139.

[5] A. Sen, Developments in Superstring Theory, hep-th/9810044.

[6] J. Maharana, Recent Developments in String Theory, hep-th/9911200.

[7] I. Bakas, Nucl. Phys. B428 (1994) 374, hep-th/9402016. 
[8] J. Maharana, Phys. Rev. Lett. 75 (1995) 205, hep-th/9502001; Mod. Phys.Lett. A11 (1996), hep-th/9502002.

[9] J. H. Schwarz, Nucl. Phys. B447 (1995) 137, hep-th/9503127; Nucl. Phys.B454 (1995) 427, hep-th/9506076; Classical Duality Symmetries in Two Dimensions, hep-th/9505170; a collection of references to earlier works can be found inthese papers.

[10] A. Sen Nucl. Phys. B447 (1995) 62, hep-th/9503057.

[11] H. Nicolai, Phys. Lett. B235 (1990) 195.

[12] V. Belinskii and V. Zakharov, Sov. Phys. JETP, 48 (1978) 985; H. Nicolai, Schladming Lectures, Springer-Verlag, Berlin (1991) eds. H. Mitter and H. Gausterer.

[13] H. Nicolai, D. Korotkin and H. Samtleben, Integrable Classical and quantum Gravity, NATO Advanced Study Institute on quantum Fields and quantum spacetime, Cargese, 1996; hep-th/9612065.

[14] P. Breitenlohner and D. Maison, Inst. H. Poincare, 46 (1987) 215; F. J. Ernst, A. Garcia and I. Hauser, J. Math. Phys. 28 (1987) 2155.

[15] A. Das, J. Maharana and A. Melikyan, Duality and Integrability of Two Dimensional String Effective Action, Phys. Lett. B533 (2002) 146, hep-th/0111158.

[16] A. Das, J. Maharana and A. Melikyan, Phys. Lett. B518 (2001) 306, hep-th/0107229.

[17] A. Das, J. Maharana and A. Melikyan, Phys. Rev. D65 (2002) 126001.

[18] C. Callan, S. Giddings, J. Harvey and A. Strominger, Phys. Rev. D45 (1992), hep-th/911105.

[19] C. Nappi and E. Witten, Phys. Lett. B293 (1992) 309, hep-th/9206078.

[20] J. Maharana and J. H. Schwarz, Nucl. Phys. B390, hep-th/9207016.

[21] S. Hassan and A. Sen, Nucl. Phys. B375 (1992) 103, hep-th/ 9109038.

[22] S. Chandrasekhar and B. C. Xanthopoulos, Proc. R. Soc. Lond. A398 (1985) 223.

[23] V. Ferrari, J. Ibanez and M Bruni, Phys. Rev. D36(1987) 1053.

[24] U. Yurtsever, Phys. Rev. D37 (1988) 2790; Phys. Rev. D38 (1988) 1706.

[25] J. B. Griffiths, Colliding Gravitational Waves, Oxford University Press.

[26] A. Feinstein, K. E. Kunze and M. A. Vazquez-Mozo, Class. Quant. Grav. 17 (2000) 3599, hep-th/0002070.

[27] V. Bozza and G. Veneziano, JHEP 0010 (2000) 035, hep-th/0007159.

[28] M. Gasperini, J. Maharana and G. Veneziano, Phys. Lett. B296 (1993) 51, hep-th/9209052.

[29] A. Sen, Nucl. Phys. B440 (1995) 421, hep-th/9411187.

[30] D. Youm, Phys. Rep. 316 (1999) 1-232, hep-th/9710046.

[31] N. Markus and J. Schwarz, Nucl. Phys. B228 (1983) 145.

[32] M. Duff and J. Lu, Nucl. Phys. B347 (1990) 394.

[33] A. Sen, Nucl. Phys. B434 (1995) 179, hep-th/9408083.

[34] M. Cvetic and D. Youm, Nucl. Phys. B472 (1996) 249, hep-th/9512127. 
[35] R. Kallosh, A. Linde, T. Ortin, A Peet and A. Van Proeyen, Phys. Rev. D46 (1992) 5278, hep-th/9205027; R. Kallosh and T. Ortin, Phys. Rev. D48 (1993) 742, hep-th/9302109 and E. Bergshoeff, R. Kallosh and T. Ortin, Phys. Rev. D50 (1994) 5188, hep-th/9406009.

[36] A. Das, J. Maharana and S. Roy, Phys. Lett. B421 (1998) 185, hep-th/9709017.

[37] P. Schwarz, Phys. Rev. D (1997) 7833, hep-th/9707233.

[38] N. Breton, T. Matos and A. Garcia, Phys. Rev. D53 (1996) 1868, hep-th/9511163. 